INPLASY

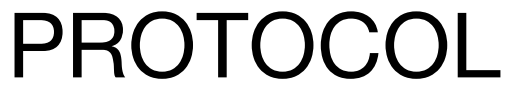

To cite: López-Valverde et al. Association of gastric infection and periodontal disease through Helicobacter pylori as a common denominator: $A$ systematic review and metaanalysis. Inplasy protocol 2021100097. doi: 10.37766/inplasy2021.10.0097

Received: 26 October 2021

Published: 26 October 2021

Corresponding author: Antonio López-Valverde

alopezvalverde@usal.es

Author Affiliation:

University of Salamanca

Support: Not funded.

Review Stage at time of this submission: Data analysis Completed but not published.

Conflicts of interest: None declared.

\section{Association of gastric infection and periodontal disease through Helicobacter pylori as a common denominator: A systematic review and meta-analysis}

López-Valverde, N1; López-Valverde, A2; Suarez, $A^{3}$; Macedo de Sousa, B4; Aragoneses, JM5;

Review question / Objective: Is gastric helicobacter pylori infection related to periodontal diseases?

Condition being studied: Therefore, the aim of this systematic review and meta-analysis was to identify and analyze clinical studies to determine the direct correlation between Helicobacter Pylori gastric infection andPeriodontal Disease. Study designs to be included: Clinical studies that provided data on Helicobacter Pylori infection in both the stomach and oral cavity, confirmed by polymerase chain reaction (PCR), rapid urease test (RUT) or enzyme-linked immunosorbent assay (ELISA). Clinical studies that associated PD with Helicobacter Pylori. The diagnosis of PD was confirmed according to the diagnostic criteria in periodontology.

INPLASY registration number: This protocol was registered with the International Platform of Registered Systematic Review and Meta-Analysis Protocols (INPLASY) on 26 October 2021 and was last updated on 26 October 2021 (registration number INPLASY2021100097).

\section{INTRODUCTION}

Review question / Objective: Is gastric helicobacter pylori infection related to periodontal diseases?

Rationale: The population $(P)$ was defined as subjects with gastric HePy infection; the intervention (I) was defined as the diagnosis of gastric infection; the comparison group (C) corresponded to subjects without gastric HePy infection; the outcome (O) was defined as the PD; and the study design (S) was defined to include non-interventional, cross-sectional, or cohort case-control studies. 
Condition being studied: Therefore, the aim of this systematic review and meta-analysis was to identify and analyze clinical studies to determine the direct correlation between Helicobacter Pylori gastric infection andPeriodontal Disease.

\section{METHODS}

Search strategy: The PubMed, EMBASE and Web of Science (WOS) databases were searched to identify articles that were eligible until September 2021. The search terms used were: [MeSH terms] 'helicobacter pylori AND periodontal diseases', [MeSH terms] 'helicobacter pylori AND chronic periodontitis' and [MeSH terms] 'helicobacter pylori AND periodontitis'. The Boolean operator "AND" was used to combine the searches. In addition, other articles were retrieved by hand searching recent reviews.

Participant or population: A total of 1,292 articles were identified up to September 2021. After exclusion, a total of 14 eligible articles were included for analysis; a total of 225,856 subjects were studied.

Intervention: Was defined as the diagnosis of gastric infection.

Comparator: The comparison group corresponded to subjects without gastric Helicobacter Pylori infection.

Study designs to be included: Clinical studies that provided data on Helicobacter Pylori infection in both the stomach and oral cavity, confirmed by polymerase chain reaction (PCR), rapid urease test (RUT) or enzyme-linked immunosorbent assay (ELISA). Clinical studies that associated PD with Helicobacter Pylori. The diagnosis of PD was confirmed ac-cording to the diagnostic criteria in periodontology.

Eligibility criteria: Cross-sectional studies, cohort studies, and case-control studies; studies published in English.

Information sources: PubMed, EMBASE and Web of Science.
Main outcome(s): To identify and analyze clinical studies to determine the direct correlation between Helicobacter Pylori gastric infection and Periodontal Dideases.

Quality assessment / Risk of bias analysis: The Newcastle-Ottawa Scale was applied to assess the quality of all studies.

Strategy of data synthesis: The metaanalysis was performed with RevMan software (Review Manager (RevMan) [Software]. Version 5.4.1, The Cochrane Collaboration, 2020).

Subgroup analysis: Studies using PCR as a method to identify helicobacer pylori in the oral cavity. Studies using RUT as a method to identify helicobacer pylori in the oral cavity.

Sensitivity analysis: Not used.

Language: English.

Country(ies) involved: Spain.

Keywords: Helicobacter pylori, gastric infection, periodontal diseases, periodontitis, chronic periodontitis.

Contributions of each author:

Author 1 - Nansi López-Valverde Conceptualization, fomal anaylsis, data curation and writing.

Email: nlovalher@usal.es

Author 2 - Antonio López-Valverde Conceptualization, validation and writing and supervision.

Email: alopezvalverde@usal.es

Author 3 - Ana Suarez - Methodology and data curation.

Email: ana.suarez@universidadeuropea.es

Author 4 - Bruno Macedo de Sousa Validation.

Email: bsousa@fmed.uc.pt

Author 5 - Juan Manuel Aragoneses Validation.

Email: jaraglam@uax.es 